\title{
Erratum to: A systems view of type 2 diabetes-associated metabolic perturbations in saliva, blood and urine at different timescales of glycaemic control
}

\author{
Noha A. Yousri ${ }^{1,2}$ • Dennis O. Mook-Kanamori ${ }^{1,3,4} \cdot$ Mohammed M. El-Din Selim $^{5}$. \\ Ahmed H. Takiddin ${ }^{5}$ Hala Al-Homsi ${ }^{5}$ Khoulood A. S. Al-Mahmoud ${ }^{5}$. \\ Edward D. Karoly ${ }^{6}$. Jan Krumsiek ${ }^{7} \cdot$ Kieu Trinh Do $^{7}$ • Ulrich Neumaier ${ }^{7}$. \\ Marjonneke J. Mook-Kanamori ${ }^{1}$ - Jillian Rowe ${ }^{1}$ - Omar M. Chidiac ${ }^{8}$. Cindy McKeon ${ }^{8}$. \\ Wadha A. Al Muftah ${ }^{1}$ • Sara Abdul Kader ${ }^{1}$. Gabi Kastenmüller ${ }^{9,10} \cdot$ Karsten Suhre $^{1}$
}

Published online: 2 July 2015

(C) Springer-Verlag Berlin Heidelberg 2015

\section{Erratum to: Diabetologia \\ DOI 10.1007/s00125-015-3636-2}

The authors regret that Kieu Trinh Do's name was spelt incorrectly in the author list. The details given in this erratum are correct.

Karsten Suhre

karsten@suhre.fr

1 Department of Physiology and Biophysics, Weill Cornell Medical College in Qatar, Qatar Foundation - Education City, PO Box 24144, Doha, Qatar

2 Department of Computer and Systems Engineering, Alexandria University, Alexandria, Egypt

3 Department of Clinical Epidemiology, Leiden University Medical Centre, Leiden, the Netherlands

4 Department of Endocrinology, Leiden University Medical Centre, Leiden, the Netherlands
Dermatology Department, Hamad Medical Corporation, Doha, Qatar

6 Metabolon Inc., Durham, NC, USA

7 Institute of Computational Biology, Helmholtz Zentrum München, German Research Center for Environmental Health, Neuherberg, Germany

8 Clinical Research Core, Weill Cornell Medical College in Qatar, Qatar Foundation - Education City, Doha, Qatar

9 Institute of Bioinformatics and Systems Biology, Helmholtz Zentrum München, German Research Center for Environment Health, Neuherberg, Germany

10 German Center for Diabetes Research (DZD), Neuherberg, Germany 